\title{
Topologieoptimierung - Entwicklungswerkzeug für die additive Fertigung
}

\author{
Alexander Walzl und Bruno Buchmayr \\ Lehrstuhl für Umformtechnik, Montanuniversität Leoben, Leoben, Österreich
}

Eingegangen 18. Januar 2017; angenommen 30. Januar 2017; online publiziert 16. Februar 2017

\begin{abstract}
Zusammenfassung: Für die Verwendung von Optimierungsmethoden (Topologie-, Topographie-, Größen- und Formoptimierung) für die additive Fertigung ist die Kenntnis über die jeweiligen Vor- und Nachteile von zentraler Bedeutung. Zusätzlich ist für eine nachfolgende Gitteroptimerung die Implementierung von Prozessparametern während der Entwicklung zu berücksichtigen. Auf dieser Grundlage wird der Nutzen der Optimierung für die additive Fertigung diskutiert. Die additive Fertigung bietet nicht nur eine große Designfreiheit für die Entwicklung neuer Bauteile sondern forciert auch einen Paradigmenwechsel von einem fertigungsgerechten Design hin zu einer Designgerechten Fertigung.
\end{abstract}

Schlüsselwörter: Additive Fertigung, DMLS, L-PBF, Topologieoptimierung, Gestaltoptimierung, Größenoptimierung, Gitterstrukturen, Bionische Strukturen, Pulverbett, Relative Dichte, Hyper Works

Topology Optimisation - Design Tool for Additive Manufacturing

Abstract: It is important for the usability of optimisation methods (topology, topography, free size, and free shape) to know the advantages and disadvantages of optimisation for additive manufacturing. Furthermore, the subsequent grid structure optimisation requires proper processing conditions during the development and construction phases. Based on these facts, the benefit of different optimisation methods for additive manufacturing is discussed. Additive manufacturing means not only design freedom, but also a paradigm shift from manufacturing-driven design to design-driven manufacturing.

Dipl.-Ing. A. Walzl (凹) · Univ. Prof. Dipl.-Ing. Dr. B. Buchmayr (凹) Lehrstuhl für Umformtechnik,

Montanuniversität Leoben

Franz-Josef-Str. 18,

8700 Leoben, Österreich

Alexander.Walzl@unileoben.ac.at;

Bruno.Buchmayr@unileoben.ac.at
Keywords: Additive manufacturing, DMLS, L-PBF, Topology optimisation, Shape optimisation, Size optimisation, Lattice structures, Bionic structures, Powder bed, Relative density, Hyper Works

\section{Einleitung}

Mit Hilfe von gängigen Optimierungsmethoden ist es heutzutage möglich, einen zielorientierten und wirtschaftlichen Leichtbau zu betreiben. Am häufigsten werden Gewichtsoder Massenreduktion als Ziele für Optimierungsaufgaben verwendet. Ein wesentlicher Grund hierfür ist der signifikante Einfluss des Materialvolumens auf die Herstellungskosten des fertigen Bauteiles. Ein weiterer Grund für die Notwendigkeit einer wirtschaftlicheren Nutzung von Materialien sind z.B. dynamisch bewegte Massen. Physikalisch betrachtet besitzen massive Bauteile eine sehr große gespeicherte kinetische Energie und führen zu großen Trägheitskräften. Im Falle eines Bauteils aus der Automobilindustrie würden massive Komponenten auch zu einer Verschlechterung des Crashverhaltens beitragen. Typische Anwendungsgebiete für eine zielorientierte Optimierung mechanischer Strukturen finden sich primär im Automobilbau und im Luft- und Raumfahrtsektor.

Durch die Optimierung werden oft abstrakte Formen generiert, die konventionell schwer oder nicht herstellbar sind. Somit ist eine Verbindung der Optimierungsmethoden mit zukunftsorientierten Fertigungstechnologien notwendig, um das volle Designpotential nutzen zu können. Die wesentlichen Vorteile der additiven Fertigung sind in der konstruktiven Designfreiheit und somit bei der Herstellung besonders komplexer Strukturen zu finden. Aus diesem Grund werden im weiteren Verlauf die Vor- und Nachteile der Optimierung sowie die Herstellung steifer und dabei besonders leichter Bauteile, unter der Berücksichtigung der Prozesseigenschaften der additiven Fertigung zusammengefasst dargestellt. Schlussendlich führt die Kopplung von Leichtbauwerkstoffen mit der numerischen Topologie-Optimierung und der additi- 
Abb. 1: Durch Topographieoptimierung optimiertes Blechbauteil. Belastung durch Punktlast und feste Einspannung der restlichen Eckknoten. Zusätzliche Implementierung von diagonalen Symmetrieebenen

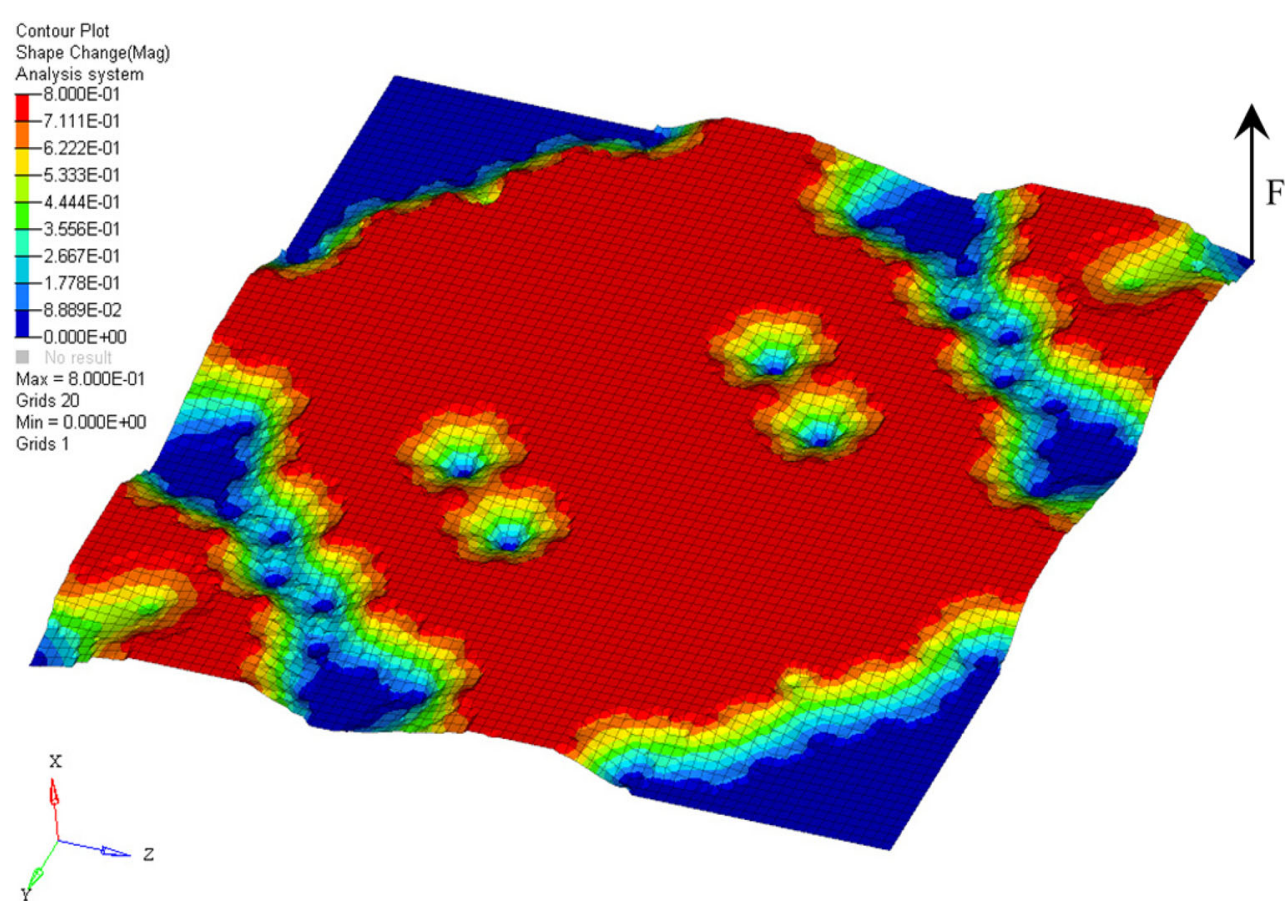

ven Fertigungstechnologie zu einer Schonung aller für die Herstellung verwendeten Ressourcen.

\section{Optimierungsmethoden}

Generell kann die Optimierung in vier grundlegende Konzepte eingeteilt werden. Diese Basiskonzepte sind die Topographie-, die Topologie-, die Größen- und Formoptimierung.

\subsection{Topographieoptimierung}

Die Topographieoptimierung basiert im Wesentlichen auf der Umgestaltung der Oberfläche von blechartigen Bauteilen. Der verwendete Algorithmus maximiert durch die Strukturierung der Oberflächenknoten den Widerstand gegen eine vordefinierte Belastung. Die Strukturierung erfolgt durch Hinzufügen von Sicken und ähnlichen Versteifungen $[1,2]$. Ein wesentlicher Vorteil dieser Optimierungsmethode ist die Entwicklung leichter und steifer Blechbauteile aus einem bestimmten Werkstoff. Zur Verdeutlichung des Potentials dieser Optimierungsmethode wird im Folgenden mit der Software HyperWorks eine numerische Fallstudie durchgeführt.

Ausgangsgeometrie der Berechung ist ein quadratisches Blechbauteil $(20 \times 20 \mathrm{~mm})$, welches durch eine Punktkraft $(10 N)$ belastet wird. Drei der vier Eckknoten werden mit einer Volleinspannung fixiert (keine Rotation und keine Translation). Die zuvor erwähnte Punktkraft wird auf den vierten Eckknoten unidirektional aufgebracht (Abb. 1). Ergebnis der Biegesimulation ist eine maximale Auslenkung von $0,98 \mathrm{~mm}$ am belasteten Eckknoten. Im Anschluss an die strukturmechanische Simulation wurde das Modell für die nachfolgende Topographieoptimierung vorbereitet. Im Allgemeinen benötigen Optimierungsaufgaben unterschiedliche Resonanzparameter, Randbedingungen und eine Zielfunktion für eine erfolgreiche Berechnung. Im vorliegenden Fall werden für eine Topographieoptimierung zwei Resonanzparameter (maximale Auslenkung und Nachgiebigkeit), eine Randbedingung (Auslenkung $<0,05 \mathrm{~mm}$ ) sowie eine Zielfunktion (minimiert die Nachgiebigkeit, um die Steifigkeit zu maximieren) dem Simulationsmodell hinzugefügt. Zur weiteren Steuerung der resultierenden optimierten Struktur werden zusätzlich zwei diagonale Symmetriebedingungen definiert. Schlussendlich wird nach einigen wenigen Iterationen ein Designvorschlag (Abb. 1) für die gewählten Parameter erhalten. Die Anpassung der Geometrie, das Hinzufügen von Sicken, resultiert in einer Reduktion der maximalen Auslenkung des Kraftangriffspunktes von ca. $56 \%$ (von $0,98 \mathrm{~mm}$ auf $0,43 \mathrm{~mm}$ )

\subsection{Topologieoptimierung}

Nach der Topographieoptimierung stellt die Topologieoptimierung die wichtigste Methode dar, um Leichtbauaspekte in die Entwicklung neuer Bauteile einfließen zu lassen. Im Vergleich zur Topographieoptimierung erzeugt die Topologieoptimierung leichte und steife „voluminöse“ Strukturen. Im Simulationsprogramm äußert sich dieser Umstand darin, dass anstatt 2D-Elementen nun 3D-Elemente verwendet werden. Für die Optimierung von Volumenkörpern ist die von C. Mattheck entwickelte SKO (=soft kill option)-Methode (GI. 1) weit verbreitet [3, 4].

$$
E_{n+1}=E_{n}+k\left(\sigma_{n}-\sigma_{r e f}\right)
$$



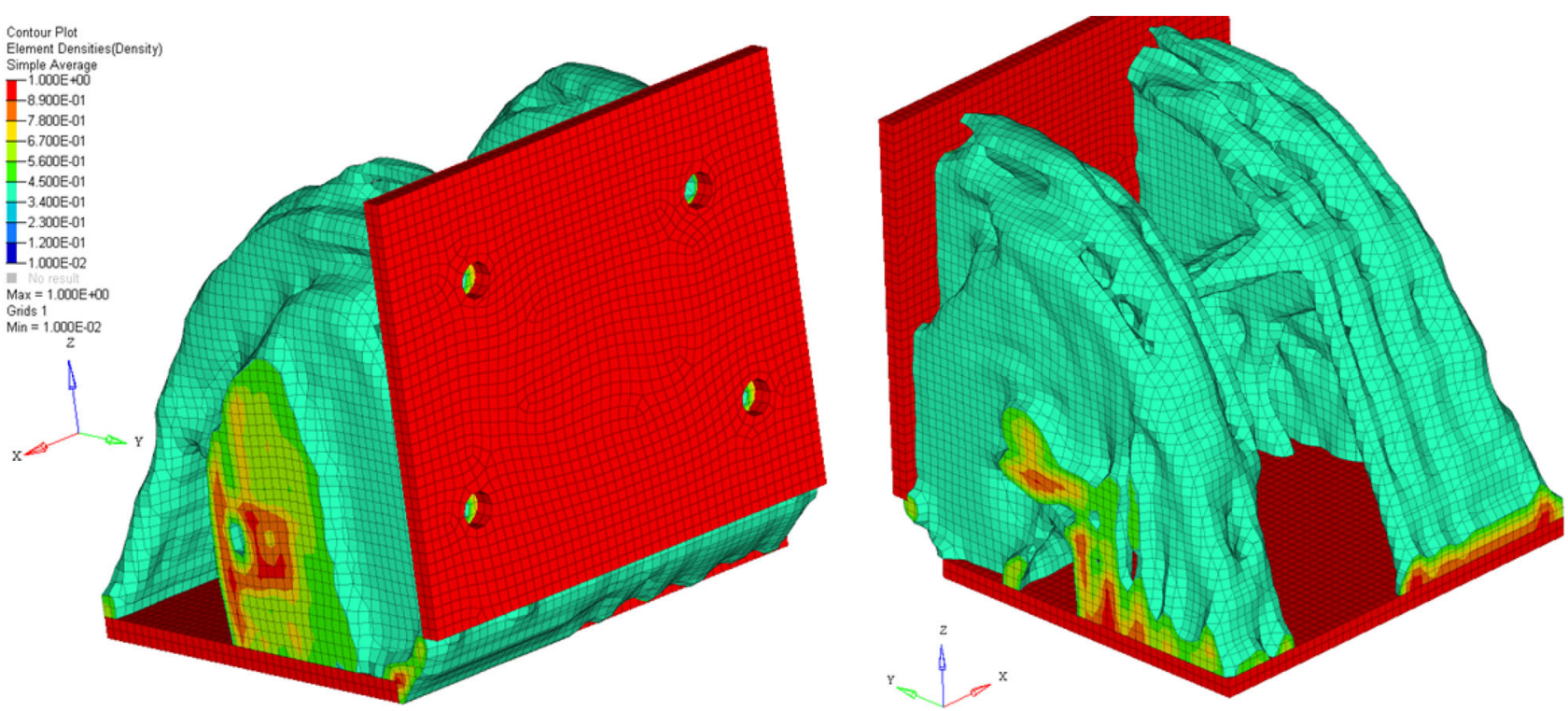

Abb. 2: Topologieoptimierte Konsole mit Strebendurchmesser- und Spannungsrestriktion. Befestigungsplatten sind voll eingespannt. Die Belastung erfolgt über vier Befestigungsbohrungen an der vertikalen Platte

Durch diesen Algorithmus (GI. 1) wird während mehrerer Iterationen der lokale Elastizitätsmodul E derart angepasst, dass die Differenz zum dem Referenz- oder Ausgangselastizitätsmodul maximal wird. Durch diese Vorgehensweise entsteht eine den Kraftpfaden folgende wurzeloder netzartige voluminöse und steife Struktur (Abb. 2; [4]).

Wie bereits beschrieben, ist die resultierende Struktur jener von natürlichen Wurzelnetzwerken sehr ähnlich. Somit beruht die Grundfunktionalität der Topologieoptimierung auf der Lokalisierung belasteter Bereiche und der anschließenden Generierung von Stützstrukturen in den identifizierten Bereichen. Über Optimierungsparameter können die Eigenschaften bzw. die Geometrie der resultierenden Wurzelstruktur beeinflusst werden. Hierfür können maximale und minimale Durchmesser sowie definierte Abstände oder Symmetrien vordefiniert werden. Zusätzlich zu diesen direkten geometrischen Parametern lässt sich auch eine Struktur entwickeln, die nicht nur für eine bestimmte Belastung "geometrisch" optimiert ist, sondern auch indirekt Werkstoffeigenschaften, wie z.B. eine zulässige Spannung, berücksichtigt. Ähnlich wie bei der Topographieoptimierung kann dies über die Definition der Optimierungsparameter softwareseitig gesteuert werden.

\subsection{Größen- und Formoptimierung}

Eine weitere Optimierungsmethode ist die so genannte Größen- und Formoptimierung. Diese Methode optimiert Geometrien durch die Verschiebung von Oberflächenknoten. Dadurch kommt eine Verdickung oder Ausdünnung von Streben oder Wänden zustande. Häufig wird diese Optimierungsmethode nach einer erfolgreichen Topologieoptimierung durchgeführt, damit das volle Potential ausgeschöpft wird [4,5]. Abb. 3 zeigt die notwendigen Iterationsschritte für die Formoptimierung.
Die Formoptimierung dient dazu, um kritische Bereiche mit hohen Spannungen zu entschärfen [6]. Im vorliegenden Fall wird eine maximale Kerbspannung reduziert und somit die Bauteillebensdauer verlängert. Das selbe Optimierungsprinzip kann auch in der Natur beobachtet werden. Viele bionische Gestaltungskonzepte basieren auf einer (Energie-)Spannungsminimierung. Ein weiteres Beispiel ist das belastungsbezogene und auch belastungsinduzierte Wachstum von menschlichen Knochen. Der Knochenaufbau besteht im Wesentlichen aus einer soliden und massiven Hülle, welche mit einem offenen Knochennetzwerk gefüllt ist. Die Dichte des Knochennetzwerks ist weiter direkt abhängig von den äußeren Belastungen. Dieses biologische Prinzip der Selbstjustierung führt dazu, dass der menschliche Knochen sich immer an die äußere Belastung anpasst und somit immer eine leichte und zugleich steife Konstruktion bildet. Ein Beispiel dazu ist die Kallusbildung bei Knochenbrüchen.

Zur Verdeutlichung der Größenoptimierung wird ein Simulationsmodell, wie in Abb. 4 dargestellt, verwendet. Hierbei werden mehrere gebogene und mit zwei Platten verbundene Rohre durch ein Drehmoment belastet. Durch die Krümmung der Rohre ist eine komplexe Spannungsverteilung zu erwarten. Im Zuge der Größenoptimierung werden die einzelnen Durchmesser der Rohre so optimiert, dass die aufgebrachte Belastung bestmöglich abgefangen und die Verdrehung minimiert werden kann. Zusätzlich kann auch eine minimale und maximale Wandstärke in der Berechung berücksichtigt werden. Das Ergebnis der Simulation ist ein Designvorschlag für variable Durchmesser über die einzelnen Rohre (Abb. 4).

Die beschriebenen Optimierungsmethoden bilden die vier Grundverfahren für die Optimierung von Bauteilen. Durch deren Hilfe kann die Entwicklung und Konstruktion von neuen Bauteilen unter der Berücksichtigung einer maximalen Materialeffizienz erfolgreich und zielgerichtet durchgeführt werden. 
Abb. 3: FormoptimierungOptimierung einer Kerbe hinsichtlich Spannungsminimierung. Die einzelnen Konturlinien zeigen die iterative Veränderung der Oberfläche im Kerbbereich $\left(\mathrm{F}_{1}<\mathrm{F}_{2}\right)$

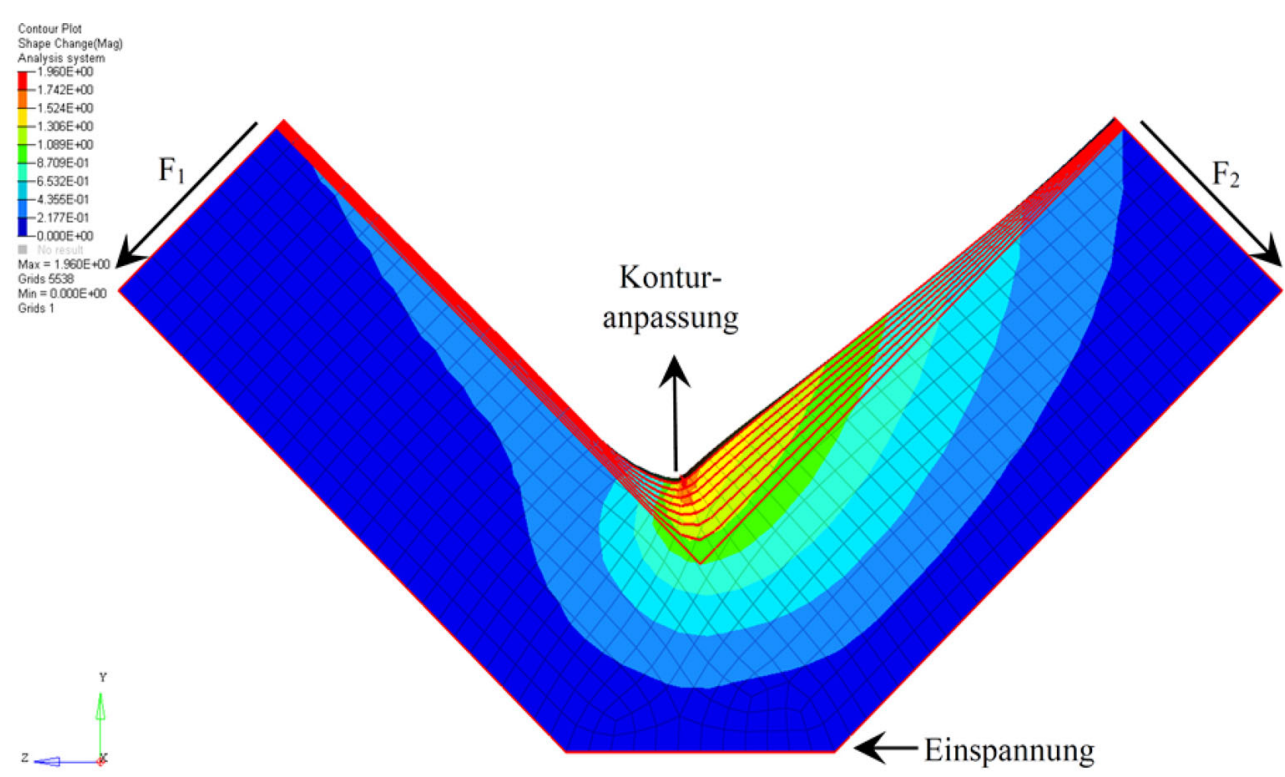

lid Isotropic Material with Penalization) Algorithmus. Seit HyperWorks V13 wird der Elastizitätsmodul E der GitterEinheitszelle über den SIMP Ansatz (GI. 2) bestimmt.

$$
E=E_{0} * \rho^{P}
$$

Hierbei wird mittels " $\mathrm{P}^{\prime}$ die resultierende Dichteverteilung und somit die Sensitivität der Dichte $\rho$ festgelegt. Die Berechnung der mechanischen Eigenschaften von beliebigen offenen oder geschlossenen Zellstrukturen wurde bereits von L. Gibson und M. Ashby $[8,9]$ ausführlich beschrieben. Mit Hilfe des dadurch erhaltenen relativen Elastizitätsmoduls kann der Optimierungsalgorithmus versorgt und die Optimierung durchgeführt werden. Neben der Berechnung des "Struktur-Elastizitätsmoduls" ist auch die Berechnung der resultierenden Zellspannung $\sigma_{N}$ erforderlich. Die resultierende Zellspannung wird über die betroffenen Zellknoten gemittelt. Für eine Anpassung der resultierenden Zellspannung wird ein variabler Parameter " $\mathrm{K}^{\prime \prime}$ in die Gleichung (GI. 3) integriert.

$$
\sigma_{N}=\left[\frac{1}{n} \sum_{i=1}^{n}\left(\frac{\sigma_{i}}{\sigma}\right)^{k}\right]^{\frac{1}{k}}
$$

Diese Ansätze bieten die Möglichkeit Gitterstrukturen und deren Anwendung im Entwicklungsprozess von Bauteilen zu integrieren. Neben den beschriebenen Vorteilen beinhaltet dieses Vorgehen auch einen großen Nachteil. Die generierten Gitter- oder Fachwerkstrukturen sind streng vom zuvor generierten FEM-Netz abhängig. Stäbe können nur innerhalb einer Zelle und zwischen zwei Elementknoten generiert werden. Somit sind nur netzabhängige (inkl. Spannungsrandbedingung) und nicht belastungsabhängige Gitterstrukturen möglich (siehe Abb. 5).

Weitere Softwarepakete für die Gitterstrukturoptimierung werden von Autodesk [10] oder Materialise [11] angeboten. Autodesk's Within bietet die Möglickeit, belastungsbezogene Gitterstrukturen zu entwickeln. Hierbei können Herstellparameter aus der additiven Fertigung so- 
Abb. 5: Optimierter Biegeträger-Topologieoptimierung und Gitterstrukturoptimierung. Gitterrestriktionen: $0,2<\rho_{\text {rel }}<0,8$. Bereiche mit einer relative Dichte $<0,2$ werden entfernt und Bereiche mit einer relativen Dichte $>0,8$ werden nicht durch Gitterstrukturen ersetzt. Die Verteilung der relativen Dichte zwischen 0,2 und 0,8 wird übervariable Strebendurchmesser erreicht

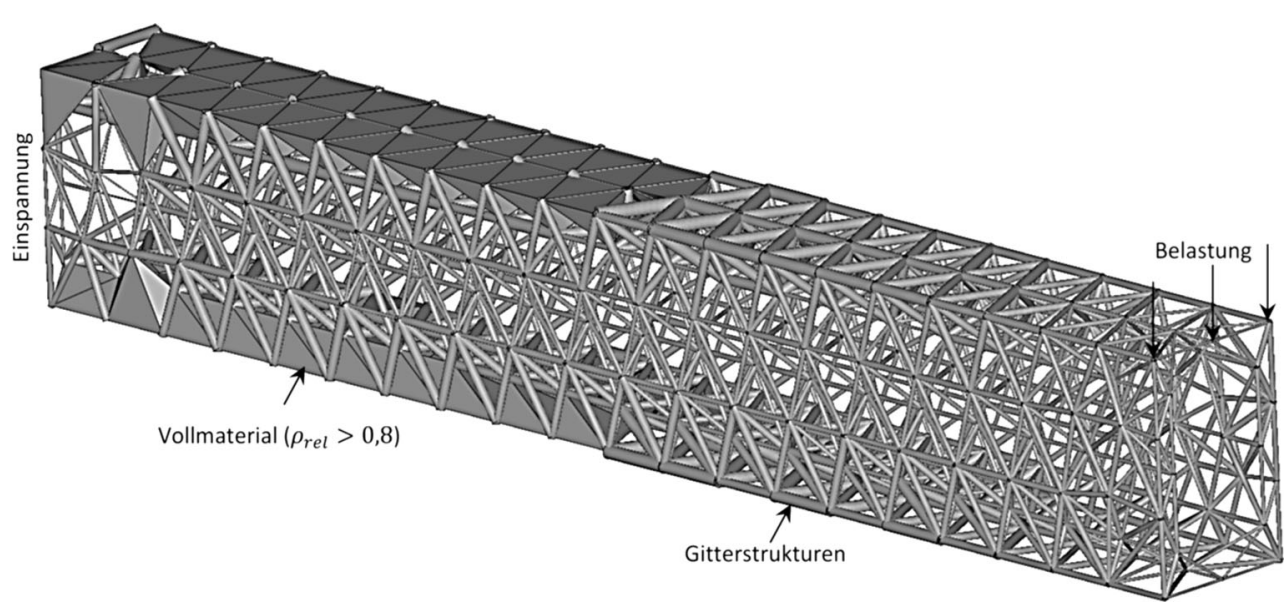

wie variable Strebendurchmesser berücksichtigt werden. Zusätzlich wird die Möglichkeit geboten nur die Oberfläche von Bauteilen zu optimieren oder zu strukturieren. Die Medizintechnik nutzt diese Technologie, um poröse und strukturierte Oberflächen auf Implantaten zu erzeugen und damit den Heilungsprozess zu beschleunigen. Somit bietet die Gitteroptimierung ein zukunftssicheres Anwendungsgebiet für die additive Fertigung - die Generierung von komplexen, sehr feinen und irregulären Strukturen. Diese Technologie steht im Wettbewerb zur üblichen Hydroxylapatit-Beschichtung für das leichtere Anwachsen des umgebenden Gewebes.

\section{Optimierungsparameter}

Jede Optimierungstechnologie benötigt nun spezifische Parameter, um zielgerichtet durchgeführt werden zu können. Im fortschreitenden Entwicklungsprozess bedeutet dies, dass man die finalen Funktionen des Bauteiles festlegt. Soll das Bauteil eine Widerstandsfähigkeit gegen Biegung, Zug oder Torsion aufweisen? Werden alle Überlegungen hinsichtlich Optimierungsparameter für jede Optmimierungstechnologie zusammengefasst, so erhält man eine übersichtliche Darstellung der Optimierungsmöglichkeiten. Das Ergebnis aus diesen Überlegungen ist in Tab. 1 zusammengefassend dargestellt.

Die Ergebnisse in Tab. 1 zeigen, dass ein Entwicklungsingenieur während der Auslegung von Bauteilen die notwendigen Optimierungsparameter berücksichtigen sollte. Andernfalls kann eine gewünschte Optmierung nicht erfolgreich angewendet werden. Zusätzlich ist die Herstellbarkeit zu berücksichtigen. Viele Designvorschläge, die mittels Topologieoptimierung generiert werden, sind durch konventionelle Fertigungsverfahren nicht herstellbar. In diesem Fall bietet meist die additive Fertigung einen Ausweg, d.h. durch das schichtartige Bauprinzip der additiven Fertigung können im allgemeinen sehr komplexe Strukturen zuverlässlich generiert werden.

\section{Nutzen der Optimierung für die additive Fertigung}

Um ein Bauteil mit einer hohen Funktionalität zu entwickeln, ist es oft notwendig, mehrere der zuvor beschriebenen Optimierungsmethoden anzuwenden. Ein Beispiel ist die Anwendung einer Topologie- und Größenoptimierung für eine Gitterstrukturoptimierung. In diesem Fall generiert die Topologieoptimierung Bereiche mit unterschiedlicher Belastung. Bereiche mit hoher Belastung bleiben solide und Bereiche mit geringerer Belastung werden in Abhängigkeit von der Belastungsintensität durch Gitterstrukturen ersetzt. Den Abschluss bildet die Kalibrierung der einzelnen Strebendurchmesser der Gitterstruktur. Dadurch werden die Gitterbereiche ebenfalls optimal auf die reduzierte Belastung abgestimmt (siehe Abb. 5). Daraus wird deutlich, dass ein derart optimiertes Bauteil konventionell nicht mehr herzustellen ist. Einzig die additive Fertigung bietet die Möglichkeit, diese Bauteile herzustellen. Die additive Fertigung, im Speziellen das Metall-Pulverbettverfahren (L-PBF), ist gekennzeichnet durch einen einzigartigen Prozessablauf. Das herzustellende Bauteil wird als CAD in viele Schichten geschnitten. Die übliche Schichtstärke für

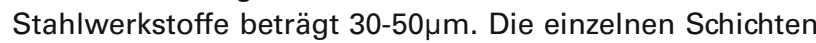
werden im Anschluss auf eine L-PBF Anlage übertragen. Nach der Einstellung der Prozessparameter, wie Laserleistung, Scangeschwindigkeit oder Füllmuster, wird eine Schicht nach der anderen von einem Laser aufgeschmolzen. Nach der Belichtung aller Schichten kann das fertige Bauteil aus der Anlage entnommen und weiter mechanisch oder thermisch behandelt werden $[12,13]$.

Analog zu den herkömmlichen Fertigungsverfahren müssen natürlich auch für die additive Fertigung prozessbedingte Einschränkungen während des Entwicklungsprozesses von Bauteilen berücksichtigt werden. Solche prozessbedingten Einschränkungen können z.B. freie Überhänge oder Hinterschnitte sowie zu schräge Wände oder geneigte Flächen darstellen. Die Standardisierung wird im Moment durch den Verein deutscher Ingenieure (VDI) sowie durch die ASTM erarbeitet. Der erste Entwurf der Norm für additive Fertigung verweist auch auf die Prüfung der relativen Dichte additiv hergestellter Bauteile. Die relative Dichte ist eine wichtige Kenngröße für die Bestimmung der 


\begin{tabular}{|c|c|c|c|}
\hline Nr. & $\begin{array}{l}\text { OPTIMIERUNGS- } \\
\text { METHODE }\end{array}$ & ANWENDUNGSBEREICH & OPTIMIERUNGSPARAMETER \\
\hline 1 & $\begin{array}{l}\text { Topologie- } \\
\text { optimierung }\end{array}$ & $\begin{array}{l}\text { Voluminöse Bauteile und Bleche } \\
\text { Die Rauheit ist abhängig von der verwendeten } \\
\text { Netzfeinheit (hex/quad vs. tet/tria) } \\
\text { Nachbearbeitung notwendig } \\
\text { Bietet Designvorschläge für die Entwicklung }\end{array}$ & $\begin{array}{l}\text { Volumenanteil, Massenanteil } \\
\text { Nachgiebigkeit, Steifigkeit } \\
\text { Versatz } \\
\text { Spannung, Dehnung } \\
\text { Schädigung } \\
\ldots\end{array}$ \\
\hline 2 & $\begin{array}{l}\text { Topographie- } \\
\text { optimierung }\end{array}$ & $\begin{array}{l}\text { Nur Blechbauteil } \\
\text { Erhöhung des Widerstandes gegen Beulen und } \\
\text { Knicken } \\
\text { Vorwiegend nur für Nichtfunktionsflächen (Sicken) } \\
\text { Steifigkeitserhöhung ohne Materialzugabe }\end{array}$ & $\begin{array}{l}\text { Lokale oder globale Spannung und Deh- } \\
\text { nung } \\
\text { Symmetrie (1-Ebene, 2-Ebenen, zyklisch, ...) } \\
\text { Breite, Länge und Schärfe von Sicken }\end{array}$ \\
\hline 3 & Formoptimierung & $\begin{array}{l}\text { Oberflächenknoten von Volumenbauteilen } \\
\text { Spannungs- und belastungsbedingte Anpassung } \\
\text { der Oberfläche } \\
\text { Reduktion von Spannungen in kritischen Bereichen } \\
\text { Gut für stufenweise Geometrieübergänge nutzbar }\end{array}$ & $\begin{array}{l}\text { Lokale Spannung oder Dehnung } \\
\text { Kerbspannung } \\
\text { Anwendung von Parameterbereichen (Obe- } \\
\text { re und untere Grenzwerte für Parameter) }\end{array}$ \\
\hline 4 & $\begin{array}{l}\text { Größen- } \\
\text { optimierung }\end{array}$ & $\begin{array}{l}\text { Dünnwandige Bauteile und Verbundwerkstoffe } \\
\text { Optimierung der Layerdicke } \\
\text { Spannungsabhängig }\end{array}$ & $\begin{array}{l}\text { Spannung, Dehnung } \\
\text { Layerdicke } \\
\text { Komposit-Layerdicke } \\
\text { Eigenfrequenz }\end{array}$ \\
\hline 5 & $\begin{array}{l}\text { Gitterstruktur- } \\
\text { optimierung }\end{array}$ & $\begin{array}{l}\text { Voluminöse Körper } \\
\text { Netzabhängige Entwicklung von Gitterstrukturen } \\
\text { Gut für die Implementierung von Leichtbauaspek- } \\
\text { ten } \\
\text { Beliebige Zelltypen möglich } \\
\text { Offene oder geschlossene Zellen möglich } \\
\text { Strukturiert } \rightarrow \text { Gitter } \\
\text { Nicht strukturiert } \rightarrow \text { Schaum }\end{array}$ & $\begin{array}{l}\text { Spannungsabhängigkeit } \\
\text { Strebendicke } \\
\text { Netzgröße (Zellgröße = Elementgröße) } \\
\text { Elementtyp (hex/quad vs. tet/tria) } \\
\text { Relative Dichte (oberer und unterer Grenz- } \\
\text { wert) } \\
\text { Schärfe der Dichteverteilung (P-Wert } \rightarrow \text { high, } \\
\text { med, low) } \\
\text { Schärfe der normierten Zellspannung } \\
\text { (k-Wert) }\end{array}$ \\
\hline
\end{tabular}

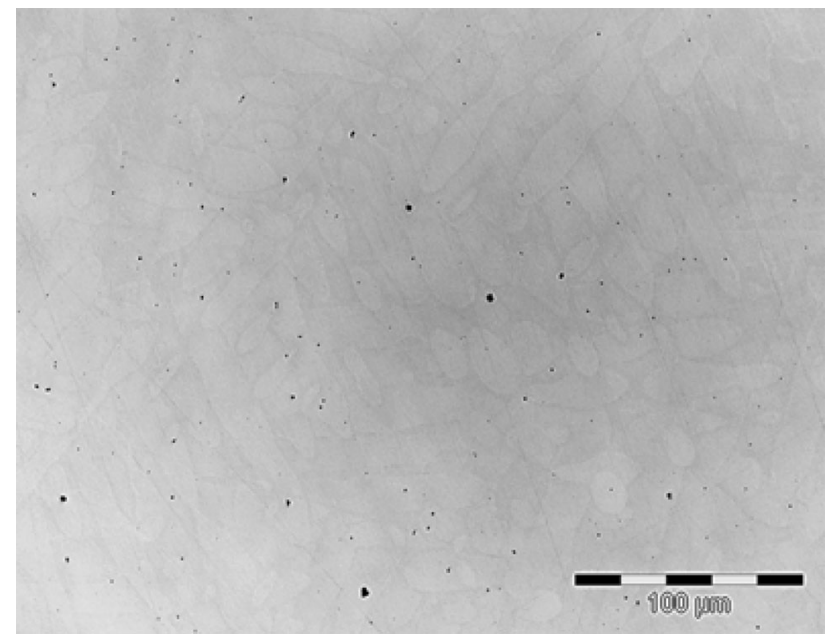

Abb. 6: Schliffbild einer AISi10Mg Probe zur Bestimmung der relativen Dichte über die Porosität. Dunkel sind die Gasporen nach dem Bauprozess dargestellt. Die relative Dichte dieser Probe beträgt 95\%

mechanischen Eigenschaften additiv gefertigter Bauteile. Speziell bei Gitterstrukturen und deren meist sehr dünnen Streben führen große Poren und Unregelmäßigkeiten im Gefüge zu einer deutlichen meist negativen Abweichung der mechanischen Eigenschaften vom Grundwerkstoff. Die erste Möglichkeit, die Porosität des Bauteiles zu bestimmen, führt über mehrere Schliffbilder. Hierfür werden von mehreren Ebenen Schliffbilder hergestellt (Abb. 6).
Im Anschluss kann durch ein Mikroskop und der zugehörigen Software der Porenanteil pro Bild bestimmt und ausgewertet werden. Zusätzlich zur optischen Methode ist es möglich, die relative Dichte $\rho_{\text {rel }}$ eines Körpers nach dem archimedischen Prinzip mit GI. 4 zu bestimmen.

$$
\rho_{\text {rel }}=\frac{\boldsymbol{m}_{\text {air }} *\left(\rho_{\mathrm{H}_{2} \mathrm{O}}-\rho_{\text {air }}\right)}{\rho_{A l} *\left(\boldsymbol{m}_{\text {air }}-\boldsymbol{m}_{\mathrm{H}_{2} \mathrm{O}}\right)}+\frac{\rho_{\text {air }}}{\rho_{A l}}
$$

Durch die Anpassung der additive Fertigungsparameter (Laserleistung, Scangeschwindigkeit, Hatchabstand oder Schichtstärke u.a.) kann die resultierende relative Dichte beeinflusst werden. Bezogen auf die Gitteroptimierung führt eine hohe relative Dichte zu einer erfolgreichen Fertigung von dünnen Gitterstrukturen mit vorhersehbaren mechanischen Eigenschaften. [14-16].

\section{Zusammenfassung und Ausblick}

Seit einiger Zeit wird die numerische Optimierung zur Entwicklung von Leichtbaustrukturen für verschiedenste Anwendungen verwendet. Die additive Fertigung bietet eine alternative Fertigungstechnologie zur Herstellung hoch komplexer, individualisierter und optimierter Bauteile. Für zukünftige Entwicklungsaufgaben müssen demnach neue Einschränkungen und Richtlinien berücksichtigt werden, 
und die Komplexität von Geometrien soll keine Grenze während der Entwicklung von Bauteilen darstellen.

Die Wichtigkeit der additiven Fertigung wird verdeutlicht durch:

- Kombination unterschiedlichster Optimierungsaufgaben, um komplexe Geometrien zu generieren

- den richtigen Einsatz dieser Optimierungsmethoden

- Verständnis für den additiven Fertigungsprozess und dessen Prozessparameter zur Beeinflussung der mechanischen Eigenschaften

- Werkstoffverständnis, um bei dünnen Strukturen gefügeneu- oder gefügeumbildende Prozesse richtig zu nutzen (z.B. In-Situ oder Post WBH)

- Designvorschläge der Optimierung nur durch die additve Fertigung herstellbar

- Implementierung von bionischen Designkonzepten

Open access funding provided by Montanuniversität Leoben.

Open Access Dieser Artikel wird unter der Creative Commons Namensnennung 4.0 International Lizenz (http://creativecommons.org/licenses/ by/4.0/deed.de) veröffentlicht, welche die Nutzung, Vervielfältigung, Bearbeitung, Verbreitung und Wiedergabe in jeglichem Medium und Format erlaubt, sofern Sie den/die ursprünglichen Autor(en) und die Quelle ordnungsgemäß nennen, einen Link zur Creative Commons Lizenz beifügen und angeben, ob Änderungen vorgenommen wurden.

\section{Literatur}

1. Klein, B.: Leichtbau-Konstruktion, Wiesbaden: Springer Fachmedien Wiesbaden, 2013

2. Reitter, G.: Leichtbau durch Sicken, http://www.4ming.de/index.php/ leichtbau-durch-sicken-fachbuch?start $=5$ (01. Mai 2016)
3. Baumgartner, A.; Harzheim, L.; Mattheck, C.: SKO (soft kill option). The biological way to find an optimum structure topology, International Journal of Fatigue, 14 (1992), Nr. 6, S. 387-393

4. Mattheck, C.; Reuschel, D.: Design nach der Natur, Physik in unserer Zeit, 30 (1999), Nr. 6, S. 253-258

5. Mattheck, C.; Bethge, K.: Zur Plausibilität der Methode der Zugdreiecke, Materialwissenschaft und Werkstofftechnik, 36 (2005), Nr. 11, S. 748-749

6. Mattheck, C.: Teacher tree. The evolution of notch shape optimization from complex to simple, Engineering Fracture Mechanics, 73 (2006), Nr. 12, S. 1732-1742

7. Altair: Hyperworks 14, http://www.altairhyperworks.de/hw14/ (01 Mai 2016

8. Gibson, L. J.: Biomechanics of cellular solids, Journal of biomechanics, 38 (2005), Nr. 3, S. 377-399

9. Gibson, L. J.; Ashby, M. F.: Cellular solids. Structure and properties, Cambridge solid state science series, Cambridge, New York: Cambridge University Press, 1999

10. Autodesk: Within,http://www.autodesk.com/products/within/ overview (01 Mai 2016)

11. Materialise: 3-matic, http://software.materialise.com/3-matic $(01$. Mai 2016)

12. Gu., D.; Meiners, W.; Wissenbach, K.; Poprawe, R.: Laser additive manufacturing of metallic components, Materials, processes and mechanisms, International Materials Reviews, 57 (2013), Nr. 3, S. $133-164$

13. Adam, G.; Zimmer, D.: Design for Additive Manufacturing-Element transitions and aggregated structures, CIRP Journal of Manufacturing, Science and Technology, 7 (2014), Nr. 1, S. 20-28

14. Ilin, A.; Logvinov, R.; Kulikov, A.; Prihodovsky, A; Xu, H.; Ploshikhin V.; Günther, B.; Bechmann, F.: Computer Aided Optimisation of the Thermal Management During Laser Beam Melting Process, Physics Procedia, 56 (2014), S. 390-399

15. Wegner, A.; Witt, G.: Correlation of Process Parameters and Part Properties in Laser Sintering using Response Surface Modeling, Physics Procedia, 39 (2012), S. 480-490

16. Krauss, H.; Zaeh, M. F.: Investigations on Manufacturability and Process Reliability of Selective Laser Melting, Physics Procedia, 41 (2013), S. 815-822 